\title{
Ultrasound reverses adriamycin-resistance in non-small cell lung cancer via positive regulation of BRAF-activated non-cod- ing RNA (BANCR) expression
}

\author{
Yong-gang Lianga, , Zhen-tian Liü,\#, Shan-xian Guo ${ }^{\mathrm{b}, *}$ \\ ${ }^{a}$ Department of Thoracic Surgery, The Second Affiliated Hospital of Nanchang University, Nanchang, Jiangxi, 330006, PR China; \\ ${ }^{b}$ Department of Internal Medicine 2, Jiangxi Cancer Hospital, Nanchang, Jiangxi, 330029, PR China;
}

\begin{abstract}
Objectives: Multi-drug resistance (MDR) remains one of the major obstacles to successful chemotherapy in patients with cancer. BRAF-activated non-coding RNA (BANCR) has been reported to be regulated in non-small cell lung cancer (NSCLC). In the present study, the researchers investigated the effects of ultrasound on BANCR expression, and its possible mechanisms were investigated in vitro and in vivo.

Methods: The Adriamycin-resistance of A549/ADM cells and the transplantation tumor model of A549/ADM in nude mice were established. Real-time PCR was used to quantify BANCR expression in cells and in mice examined with or without ultrasound. P-gp and MRP protein expression were measured by western blot. The cell viability was detected by MTT assay.

Results: ANCR expression was significantly elevated by ultrasound in A549/ADM cells and tumor tissue from xenograft mice. In in vitro experiments, P-gp and MRP levels were markedly reduced by ultrasound. When the cancer cells up-regulated BANCR by transfection of pCDNA-BANCR, P-gp and MRP levels were inhibited. In addition, the down-regulation of BANCR by si-BANCR transfection elevated their expression.

Conclusion: Ultrasound reversed adriamycin-resistance in A549/ADM cells by regulating BANCR expression, and P-gp and MRP regulation were involved in this process.
\end{abstract}

Keywords: Ultrasound; multi-drug resistance; lung cancer; IncRNAs

\section{INTRODUCTION}

Non-small cell lung cancers (NSCLCs) account for 85\% of lung cancer cases ${ }^{[1]}$. They are the most common of all lung cancer types and represent the leading cause of cancer deaths worldwide ${ }^{[2]}$. Multi-drug resistance (MDR) remains one of the major obstacles to successful chemotherapy, with more than $90 \%$ of tumor patients dying of MDR ${ }^{[3]}$. Consequently, there is still an urgent need to explore viable strategies to overcome MDR. Ultrasound, a form of energy, has been applied to the ablation and reversal of tumor cells ${ }^{[4]}$. In addition, ultrasound is reported to be a safer and more effective method for reversal of MDR in tumor cells ${ }^{[5,6]}$. However, the molecular mechanism of ultrasound treatment on cancers is still not fully understood.

\#These authors contributed equally to this work.

*Corresponding author: Shan-xian Guo

Mailing address:Department of Internal Medicine 2, Jiangxi

Cancer Hospital, 519 Beijing East Road, Nanchang, Jiangxi

Province, 330029, PR China.

E-mail : guoshanxian0830@126.com

Received: 20 October 2017 Accepted: 25 December 2017
Long non-coding RNAs (lncRNAs) are non-protein coding transcripts longer than 200 nucleotides that take part in the regulation of gene transcription and expression ${ }^{[7]}$. Emerging evidence indicates that lncRNAs are involved in the complex network of tumors and play important roles in tumorigenesis and progression [8, 9]. BRAF-activated non-coding RNA (BANCR)-a 693bp IncRNA on chromosome 9-has been reported to regulate tumor cell proliferation and migration ${ }^{[10,11]}$. A recent study found that BANCR was dysregulated in the tumor tissues of lung cancers and non-small cell lung cancers ${ }^{[12,13]}$. Specifically, the BANCR expression was closely related to the stages of tumor development.

P-glycoprotein (P-gp), also known as multidrug resistance protein 1 (MDR1), is an important protein of the cell membrane that pumps many foreign substances out of cells and is thus involved in MDR [14]. Multidrug resistance-associated protein (MRP) is another membrane glycoprotein implicated in MDR ${ }^{[15]}$. The researchers hypothesize that P-gp and MRP expression is regulated by BANCR, which is related to the reversal of MDR in NSCLC. In the present study, the 
hypothesis was proved in vitro and in vivo.

\section{MATERIALS AND METHODS}

Establishment of A549/ADM cells

The NSCLC adenocarcinoma cell lines A549 were obtained from The Second Affiliated Hospital of Nanchang University. The cells were cultured in RPMI 1640 supplemented with $10 \%$ fetal bovine serum (Gibco, USA), $100 \mathrm{U} / \mathrm{ml}$ of penicillin, and $100 \mathrm{mg} / \mathrm{ml}$ of streptomycin (Sigma, USA) at $37^{\circ} \mathrm{C}$ with $5 \%$ CO2. The logarithmic phase cells were harvested, and a singlecell suspension $(1 \times 107 / \mathrm{ml})$ was prepared. To establish the drug resistance of cells, different concentrations $(0.001 \mu \mathrm{g} / \mathrm{ml}-1.0 \mu \mathrm{g} / \mathrm{ml})$ of Adriamycin (ADM) were supplemented in the RPMI-1640 medium to incubate with cells at $37^{\circ} \mathrm{C}$ for 120 days. The medium was replaced every three days. This kept the cells growing in medium at a concentration of $1.0 \mu \mathrm{g} / \mathrm{ml} \mathrm{ADM}$ and considered to be A549/ADM cells.

Establishment of the transplantation tumor model of A549/ADM in nude mice

The researchers harvested the logarithmic phase A549/ADM cells and prepared the cell suspension with normal saline at a concentration of $1 \times 107$ cells $/ \mathrm{ml}$. Thirty-two male BALB/C-nu/nu nude mice aged four to six weeks were purchased from the Chinese Academy of Animal Medical Sciences Laboratory (Beijing, China). All the mice were anesthetized with $430 \mathrm{mg} / \mathrm{kg}$ of chloral hydrate first. Then, $150 \mu \mathrm{l}$ A549/ADM of cell suspension were injected subcutaneously into the right forelimb armpit of each mouse. The injection lasted for eight days, and all mice were housed under SPF conditions. After the model establishment, the tumor volume was measured by vernier caliper and calculated according the following formula: $\mathrm{V}=$ length $\times$ width $2 / 2$.

\section{MTT assay}

The cell viability was detected by MTT assay using an MTT Cell Proliferation and Cytotoxicity Assay Kit (Beyotime, China) according to the manufacturer's instructions. The RPMI-1640 medium without cells was considered as the control.

\section{Plasmid generation and cell transfection}

The BANCR was synthesized and cloned into the pCDNA3.1 (Invitrogen, Shanghai, China) vector. Ectopic expression of BANCR was achieved through pCDNABANCR transfection, with an empty pCDNA3.1 vector used as a control. Plasmid vectors with or without
BANCR were prepared using PureLink® HiPure Plasmid (Life Technologies, USA) and transfected into A549/ADM cells. The siRNA-BANCR (si-BANCR) and siRNA-negative control (si-control) were prepared by Hanbio Co., Ltd (Shanghai, China). The si-BANCR and si-control were transfected into A549/ADM cells using a Lipofectamine 2000 (Invitrogen, USA). Forty-eight hours after the transfection, the cells were harvested for further detection.

\section{RNA extraction and real-time PCR}

Total RNA of cells or tumor tissues was isolated with TRIzol reagent (Invitrogen, USA) according to the manufacturer's instructions. RNA was reverse transcribed to cDNA using the ImProm-II ${ }^{\mathrm{TM}}$ Reverse Transcription System (Promega, USA). The BANCR expression was quantified by an Applied Biosystems 7900HT Fast Real-Time PCR System with Power SYBR $\AA$ Green PCR Master Mix (Applied Biosystems, USA). GAPDH was used to normalize the BANCR expression. All specific primers were synthesized by Shanghai Generay Biotech Co., Ltd (Shanghai, China).

\section{Western blot}

The A549 cells or A549/ADM cells were washed once in ice-cold PBS and lysed with RIPA Lysis Buffer (Beyotime, China). The total protein of cells was abstracted using a Nuclear and Cytoplasmic Protein Extraction Kit (Beyotime, China), and protein concentration was quantified using a BCA Protein Assay Kit (Beyotime, China), so that $40 \mu \mathrm{g}$ of total protein were separated by $10 \%$ SDS gel electrophoresis (SDSPAGE), then transferred to nitrocellulose membranes (Millipore, USA) and incubated with primary antibodies (Cell Signaling Technology, USA). The horseradish peroxidase (HRP)-conjugated secondary antibody was

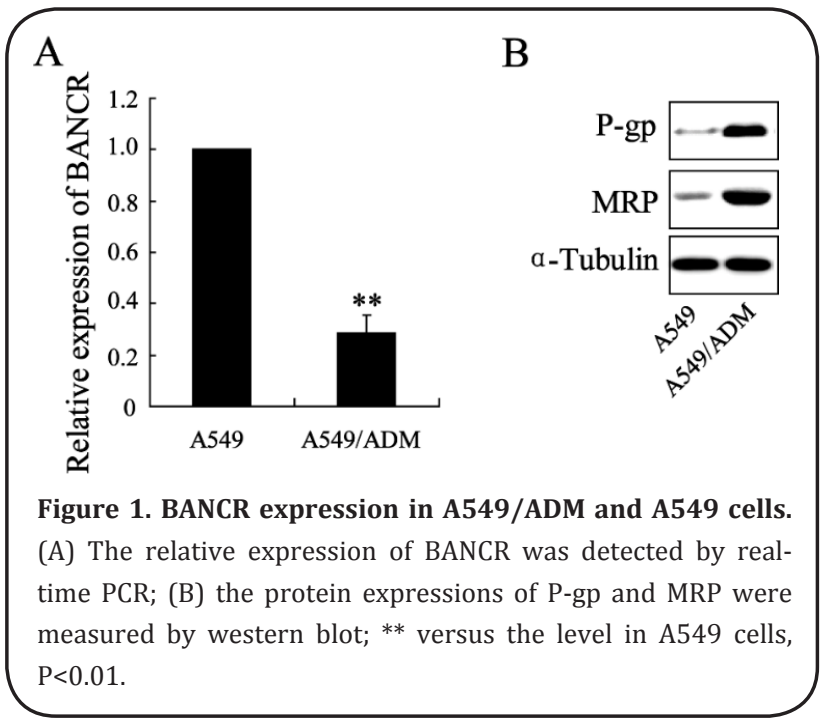


A

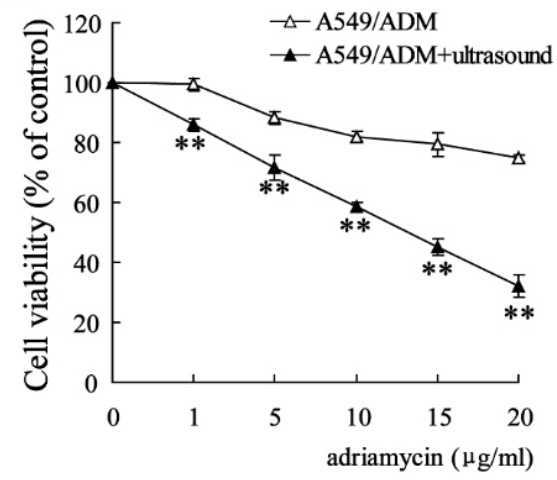

B

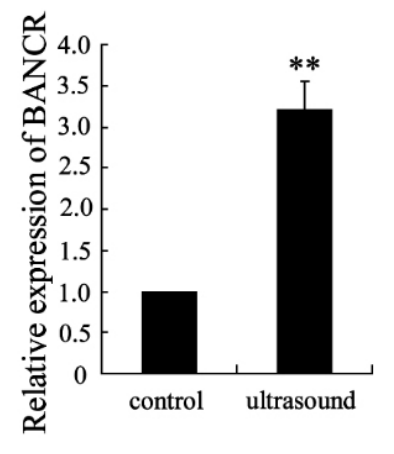

$\mathrm{C}$

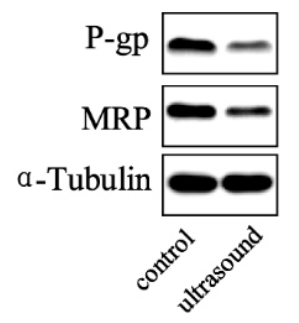

Figure 2. The effect of ultrasound on cell viability and BANCR expression of A549/ADM cells. The A549/ADM cells were subjected to an ultrasound of $0.8 \mathrm{MHz}$, pulse ratio $60 \%$, and $0.4302 \mathrm{~W} / \mathrm{cm} 2$ sound intensity for one minute; (A) the cell viability was detected by MTT assay; (B) the relative expression of BANCR was quantified by real-time PCR; (C) the protein expressions of P-gp and MRP were detected by western blot with $\alpha$-Tubulin as control; ** versus A549/ADM cells without ultrasound treatment, $\mathrm{P}<0.01$.

incubated with membranes at room temperature for one hour. The bands were visualized using enhanced chemiluminescent (ECL) substrate. Protein loading was normalized by $\alpha$-Tubulin.

\section{Statistical analysis}

All statistical analyses were performed using SPSS 18.0 software. The measurement data were presented as mean \pm standard deviation. An independent t-test was used to compare the difference between two groups. One-way ANOVA followed by Student's t-test was conducted to compare the difference between any two means. Two-tailed $\mathrm{P}$ values of $<0.05$ were regarded as statistically significant.

\section{RESULTS}

\section{BANCR expression in A549/ADM and A549 cells}

The researchers first detected the relative expression of BANCR in A549/ADM and A549 cells. As shown in Figure 1A, expression was significantly lower in A549/ ADM cells than in A549 cells. In addition, protein expressions of P-gp and MRP were markedly higher in A549/ADM cells (Figure 1B).

The effect of ultrasound on cell viability and BANCR expression of A549/ADM cells

After A549/ADM cells were subjected to ultrasound for one minute, the cell viabilities were significantly decreased, and this decrease was enhanced with the increase of Adriamycin concentrations (Figure 2A). The relative expression of BANCR in cells was elevated after ultrasound treatment (Figure 2B). In addition, ultrasound down-regulated P-gp and MRP protein expression (Figure 2C).

BANCR level regulates P-gp and MRP expression in A549/ADM cells

To overexpress the BANCR level of A549/ADM cells, the proteins of P-gp and MRP were significantly decreased (Figure 3A). In addition, to inhibit the expression of BANCR, the proteins of P-gp and MRP were markedly increased (Figure 3B).

The effect of BANCR down-regulation on A549/ADM cell viability

Si-BANCR was used to down-regulate BANCR expression of A549/ADM cells. As shown in Figure 4, the cell viability was significantly elevated by downregulation of BANCR.

The effect of ultrasound on tumor growth and BANCR expression in vivo

The model of transplantation for A549/ADM tumors in nude mice was established. After the mice were administered different treatments, the results showed that $5 \mathrm{mg} / \mathrm{kg}$ of Adriamycin alone had no effect on tumor volume, whereas tumor volume was significantly reduced by ultrasound treatment. In addition, tumor volume was markedly lower after combination therapy with Adriamycin and ultrasound than after ultrasound treatment alone (Figure 5A). The real-time PCR data indicate that ultrasound significantly elevated BANCR expression in tumor tissue, and its level of expression was even higher in the tumor treated with combination therapy (Figure 5B).

\section{DISCUSSION}


A

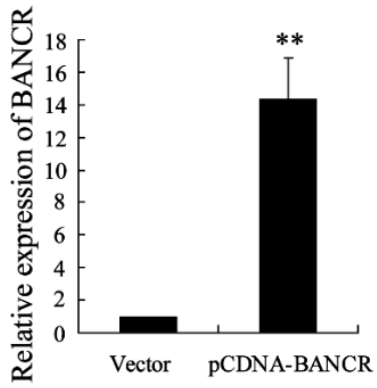

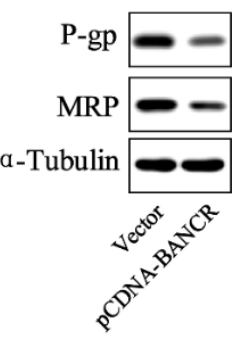

B

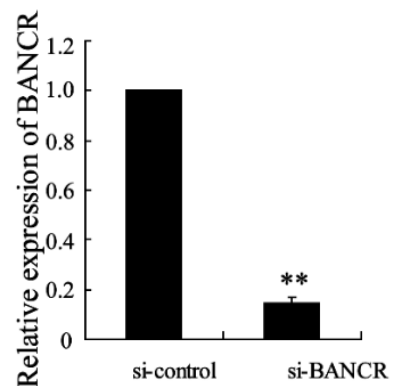

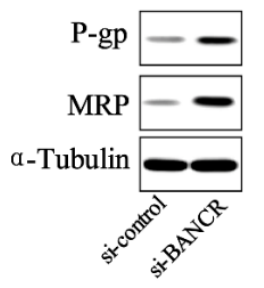

Figure 3. BANCR level regulates P-gp and MRP expression in A549/ADM cells. (A) The BANCR level was up-regulated by pCDNABANCR, and P-gp and MRP expression were measured by western blot; (B) the BANCR level was down-regulated by si-BANCR, and the P-gp and MRP expression were measured by western blot; ${ }^{* *}$ versus A549/ADM cells without ultrasound treatment, $\mathrm{P}<0.01$.

LncRNAs, a newly discovered class of noncoding genes, have been shown in the past few years to be involved in epigenetics, transcription, and post-transcriptional regulation ${ }^{[16]}$. The role of lncRNAs in gene regulatory processes is important in various human diseases, especially in cancer development ${ }^{[17]}$. Although many studies have demonstrated the functions of lncRNAs, the involvement of lncRNAs in MDR of non-small cell lung cancer is still not fully understood. In the present study, the researchers found that A549/ADM cells had lower levels of BANCR expression than A549 cells, which implies that BANCR might take part in the regulation of tumor development with MDR.

Ultrasound has been reported to be an effective approach to reversing MDRin tumor cells. Low-intensity ultrasound can result in a series of biological reactions in the histiocytes, including the alteration of material motion, volume, and endochylema flow. Previous studies have demonstrated that ultrasound reduced tumor growth, and the combination of ultrasound with chemotherapeutics could enhance antitumor effects $[18,19]$. In the present study, the researchers found that ultrasound significantly decreased the viabilities of Adriamycin-resistant A549/ADM cells in vitro and lowered the tumor volume of A549/ADM induced xenograft mice in vivo.

The researchers also investigated the effect of ultrasound on BANCR expression. The real-time PCR data indicate that BANCR levels were markedly elevated by ultrasound treatment in both A549/ADM cells and in mice, suggesting that BANCR regulation was involved in the ultrasound treatment of NSCLC tumors. In a previous study, Sun et al ${ }^{[13]}$. demonstrated that BANCR expression was reduced and associated with larger tumor size, advanced pathological stage, metastasis distance, and shorter duration of overall survival in NSCLC patients. They also found that knockdown of BANCR expression promoted cell migration and invasion in vitro. Jiang et al ${ }^{[12]}$. showed that BANCR levels were down-regulated in lung cancer cells, and the overexpression of BANCR could suppress tumor growth.

P-gp and MRP - both of which are ATP-binding cassette transporters-are ATP-dependent drug efflux pumps for xenobiotic compounds with broad substrate specificity. The overexpressions of P-gp and MRP are thought to be mechanisms in MDR development ${ }^{[14,20]}$. In the present study, the researchers also found higher levels of P-gp and MRP protein expression in A549/ ADM cells than in A549 cells. In addition, reduced expressions of P-gp and MRP were observed in cells treated with ultrasound. Within in vitro experiments, the overexpression of BANCR could decrease the levels of P-gp and MRP, whereas the down-regulation of BANCR may increase their expression. Therefore, it was believed that BANCR played a role in A549/ADM cell viability, probably via regulation of P-gp and MRP expression.

In conclusion, the expression of BANCR was markedly

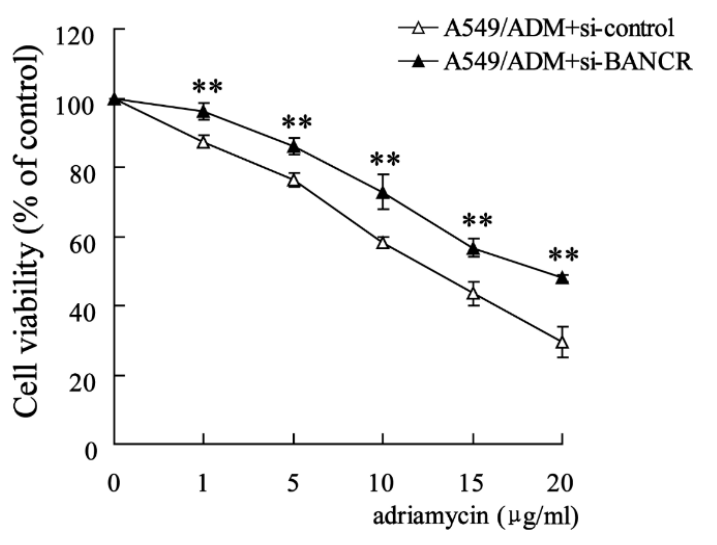

Figure 4. The effect of BANCR down-regulation on A549/ ADM cell viability. The cell viability was detected by MTT assay. ** versus A549/ADM + si-control group, $\mathrm{P}<0.01$ 

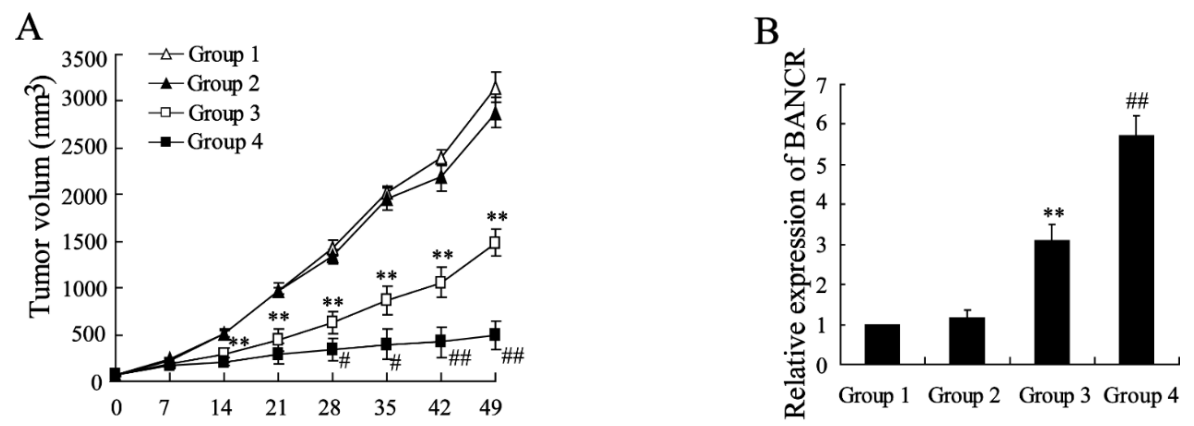

Figure 5. The effect of ultrasound on tumor growth and BANCR expression in vivo. Group 1: Tail vein injection with phosphate buffer; Group 2: Tail vein injection with 5mg/kg of Adriamycin; Group 3: The tumor was treated with ultrasound therapy; Group 4: The mice were treated with a tail vein injection of $5 \mathrm{mg} / \mathrm{kg}$ Adriamycin and ultrasound therapy; (A) The tumor volume was recorded at the beginning of treatment; (B) the BANCR expression was quantified by real-time PCR; ** versus Group 1, $\mathrm{P}<0.01$; \# versus Group 3, $\mathrm{P}<0.05$; \#\# versus Group $3, \mathrm{P}<0.01$.

down-regulated in Adriamycin-resistant NSCLC adenocarcinoma cell lines and A549 cells. Ultrasound treatment could elevate BANCR expression in A549/ ADM cells and in xenograft mice, with inhibition of P-gp and MRP expression as potential mechanisms. This study promotes understanding of the pathogenesis of NSCLC development and the mechanism of ultrasound treatment for cancer. These findings are also significant for anti-cancer therapies that employ BACNR regulation. However, the molecular mechanisms underlying the effects of IncRNAs on cancers must be further investigated.

\section{CONFLICTS OF INTEREST}

All authors declare that they have no competing interests.

\section{REFERENCES}

1. Iyer, S., Roughley, A., Rider, A., and Taylor-Stokes, G. (2014) The symptom burden of non-small cell lung cancer in the USA: a real-world crosssectional study. Supportive care in cancer : official journal of the Multinational Association of Supportive Care in Cancer 22, 181-187

2. Siegel, R., Ma, J., Zou, Z., and Jemal, A. (2014) Cancer statistics, 2014. CA: a cancer journal for clinicians 64, 9-29

3. Chen, Y. T., Feng, B., and Chen, L. B. (2012) Update of research on drug resistance in small cell lung cancer chemotherapy. Asian Pacific journal of cancer prevention : APJCP 13, 3577-3581

4. Wang, T. Y., Choe, J. W., Pu, K., Devulapally, R., Bachawal, S., Machtaler, S., Chowdhury, S. M., Luong, R., Tian, L., Khuri-Yakub, B., Rao, J., Paulmurugan, R., and Willmann, J. K. (2015)
Ultrasound-guided delivery of microRNA loaded nanoparticles into cancer. Journal of controlled release : official journal of the Controlled Release Society 203C, 99-108

5. Marin, A., Sun, H., Husseini, G. A., Pitt, W. G., Christensen, D. A., and Rapoport, N. Y. (2002) Drug delivery in pluronic micelles: effect of high-frequency ultrasound on drug release from micelles and intracellular uptake. Journal of controlled release : official journal of the Controlled Release Society 84, 39-47

6. Jiang, M. D., Peng, Z. P., Li, S. L., Wang, Z. G., Ran, H. T., Huo, S. H., and Yin, X. L. (2006) [Reversion of multidrug resistance of hepatocellular carcinoma by antisense oligonucleotides and ultrasonic microbubble intensifier transfection combined with ultrasound irradiation]. Zhonghua gan zang bing za zhi = Zhonghua ganzangbing zazhi $=$ Chinese journal of hepatology 14, 341-345

7. Mercer, T. R., Dinger, M. E., and Mattick, J. S. (2009) Long non-coding RNAs: insights into functions. Nature reviews. Genetics 10, 155-159

8. Zhang, H., Chen, Z., Wang, X., Huang, Z., He, Z., and Chen, Y. (2013) Long non-coding RNA: a new player in cancer. Journal of hematology \& oncology 6,37

9. Nie, F. Q., Zhu, Q., Xu, T. P., Zou, Y. F., Xie, M., Sun, M., Xia, R., and Lu, K. H. (2014) Long non-coding RNA MVIH indicates a poor prognosis for non-small cell lung cancer and promotes cell proliferation and invasion. Tumour biology : the journal of the International Society for Oncodevelopmental Biology and Medicine 35, 7587-7594

10. Li, R., Zhang, L., Jia, L., Duan, Y., Li, Y., Bao, L., and Sha, N. (2014) Long non-coding RNA BANCR promotes proliferation in malignant melanoma by regulating MAPK pathway activation. PloS one 9, 


\section{e100893}

11. Wang, Y., Guo, Q., Zhao, Y., Chen, J., Wang, S., Hu, J., and Sun, Y. (2014) BRAF-activated long noncoding RNA contributes to cell proliferation and activates autophagy in papillary thyroid carcinoma. Oncology letters 8, 1947-1952

12. Jiang, W., Zhang, D., Xu, B., Wu, Z., Liu, S., Zhang, L., Tian, Y., Han, X., and Tian, D. (2015) Long noncoding RNA BANCR promotes proliferation and migration of lung carcinoma via MAPK pathways. Biomedicine \& pharmacotherapy $=$ Biomedecine \& pharmacotherapie 69, 90-95

13. Sun, M., Liu, X.-H., Wang, K.-M., Nie, F.-q., Kong, R., Yang, J.-s., Xia, R., Xu, T.-P., Jin, F.-Y., Liu, Z.-J., Chen, J.-f., Zhang, E.-B., De, W., and Wang, Z.-X. (2014) Downregulation of BRAF activated non-coding RNA is associated with poor prognosis for nonsmall cell lung cancer and promotes metastasis by affecting epithelial-mesenchymal transition. Molecular cancer 13, 68-68

14. Han, L., Wang, Y. F., Zhang, Y., Wang, N., Guo, X. J., Yang, J. K., Wang, K. P., Liu, S. N., Fan, Q. X., Li, K., Jiang, J. H., and Wang, Q. D. (2012) Increased expression and function of P-glycoprotein in peripheral blood CD56+ cells is associated with the chemoresistance of nonsmall-cell lung cancer. Cancer chemotherapy and pharmacology 70, 365-372

15. Binaschi, M., Supino, R., Gambetta, R. A., Giaccone,
G., Prosperi, E., Capranico, G., Cataldo, I., and Zunino, F. (1995) MRP gene overexpression in a human doxorubicin-resistant SCLC cell line: alterations in cellular pharmacokinetics and in pattern of cross-resistance. International journal of cancer. Journal international du cancer 62, 8489

16. Gibb, E. A., Brown, C. J., and Lam, W. L. (2011) The functional role of long non-coding RNA in human carcinomas. Molecular cancer 10, 38-38

17. Harries, L. W. (2012) Long non-coding RNAs and human disease. Biochemical Society transactions 40, 902-906

18. Miller, D. L., and Song, J. (2003) Tumor growth reduction and DNA transfer by cavitationenhanced high-intensity focused ultrasound in vivo. Ultrasound in medicine \& biology 29 , 887 893

19. Mohamed, M. M., Mohamed, M. A., and Fikry, N. M. (2003) Enhancement of antitumor effects of 5-fluorouracil combined with ultrasound on Ehrlich ascites tumor in vivo. Ultrasound in medicine \& biology 29, 1635-1643

20. Hu, Y., Li, C., Li, H., Li, M., and Shu, X. (2014) Resveratrol-mediated reversal of tumor multidrug resistance. Current drug metabolism 15, 703710 